\title{
Brief Analysis about Pragmatic Function of Pun in English Advertising Based on Grice Cooperative Principle
}

\author{
Lan Zheng \\ Xi'an Peihua University, Shaan xi 710100, China \\ 747481089@qq.com
}

\begin{abstract}
English advertisement is characterized by the diversity of its forms and the flexibility and originality of its language usage, its lexical and syntactic levels are considered as distinguished features, advertising English is also frequently employed rhetorical vehicles which are used as decorative devices of advertisement language, English advertising rhetoric commonly used different techniques to enhance the attraction and appeal, including puns, for its simple, lively, humorous features, it is widely used in English advertising. Puns can produce humor, express the advertisers' wit, then enhance the appeal of language, and to attract people's attention; what's more, the use of punning in English advertising can better promote economic development and stimulate consumer consumption. At present, many scholars at home and abroad have studied puns in English advertising, including the pragmatic functions and translation methods in English advertising. According to Grice Cooperative principle---make your conversational contribution is required at the stage at which it occurs, by the accepted purpose or direction of the talk exchange in which you are engaged. This article will analyses the pragmatic functions of puns in English advertisementsin combining advertising instances with the application of cooperative principle the paper.
\end{abstract}

Keywords: English advertisements; puns; cultural background.

\section{Introduction}

With the rapid development of the advertising industry, the importance of modern advertising has been greatly increased in international social activities. Advertising is everywhere in modern life and plays an important role and deeply integrated into everyday life. As one of the carriers of information transmission, advertising is a way of disseminating information to the public, and the advertising language plays an important and indispensable role, especially in commercial activities. For commercials, the aim is to catch the eye of consumers and stimulate their desire to buy. For public service advertising, the aim is to impress and resonate with the public. In order to attract the public and have a strong attraction and influence, advertising must use effective rhetoric to enhance its language appeal, which must use puns. English advertisement is characterized by the diversity of its forms and the flexibility and originality of its language usage, its lexical and syntactic levels are considered as distinguished features, advertising English is also frequently employed rhetorical vehicles which are used as decorative devices of advertisement language, English advertising rhetoric commonly used different techniques to enhance the attraction and appeal, including puns, pun is a clever and amusing use of a word with more than one meaning, or a word that sounds like another word, so that what you say has two different meanings, since its simple, lively, humorous features, it is widely used in English advertising. Puns can produce humor, express the advertisers' wit, then enhance the appeal of language, and to attract people's attention; what's more, the use of punning in English advertising can better promote economic development and stimulate consumer consumption. In general, the advertising is generalized notification and persuasion, and the main purpose is to promote a certain product, service or concept. There is a lot of research on puns in English advertising language. Among them, Meng Lin and Zhan Jinghui mainly analyze the application techniques and translation of puns in English advertisements from four aspects: homophone pun, homophobic pun, grammar pun and idiom pun. Yao Jun mainly analyzes the characteristics and functions of puns in English advertising from a pragmatic point of view. The use of puns from the perspective of relevant pragmatics, such as Yu Mingxuan. James Brown pointed out that: "a semantic achievement and derives from the symbolic nature of language", which must be embedded with at least two meanings differ significantly from each other. Furthermore, he adds one more condition to the description of 
puns perceptions, which requires forehead knowledge of the multiple meanings of the pun word to guarantee that the puns can be successfully perceived by the targeted readers. This paper will combine the example of advertising, the use of the principle of cooperation principle to analyze the pragmatic functions of puns in English advertising.

\section{Grice Cooperative Principle}

Grice proposes the cooperative principle in 1975, which means the two parties must work together, and adhere to certain guidelines effectively and reasonably, so that what you say is consistent with the recognized purpose or direction of the conversation you are involved in, so the conversation can go smoothly. Grice further proposes four corresponding guidelines:

A. The Maxim of Quantity: make your contribution as informative as is required (for the current purpose of the exchange); Do not make your contribution more informative than is required.

B. The Maxim of Quality: try to make your contribution one that is true; Do not say what you believe to be false and do not say that for which you lack adequate evidence.

C. The Maxim of Relation: be relevant.

D. The Maxim of Manner: be perspicuous; Avoid obscurity and ambiguity; Be brief (avoid unnecessary prolixity) and orderly.

Grice points out that the importance of these principles is different, and when observing these principles, different speakers have different emphasis on different occasions, and even in order to fulfill special communicative purposes, users try to violate certain principles deliberately sometimes, thus creating "conversational meaning", which is the implicit meaning of discourse. In communication, the speaker will deliberately fail to comply with a certain principle, that is, the speaker knows that he has violated a certain principle, and let the listener know that the speaker has violated that principle, but the purpose is not to stop the conversation, but to send another message to the listener - the meaning of the conversation. The listener will also derive the meaning of the conversation based on these four guidelines. In advertising, advertisers use rhetorical techniques (including puns) to deliberately violate the principle of cooperation in communication when designing advertising language, in order to get consumers to think and use conversational guidelines to derive the deep meaning that advertising wants to convey. Language is the product of society and the crystallization of human history and culture. It condenses all the unique characteristics of human society, such as the social consciousness, history, culture, and customs and so on, which are passed down by a nation. Different cultural backgrounds and cultural traditions, there are considerable cultural differences between Chinese and western countries in the way of thinking, values, code of conduct and lifestyle and other aspects. Language is the carrier of culture, and directly reflects the differences of culture.

\section{Pragmatic Function of Puns in English Advertising}

Pun is a special language for the purpose of promoting and stimulating consumption. It can make advertisement content more exciting, more attractive, more competitive, and it is the key and core to a successful advertisement. Because the pun language is concise, pragmatic pun and vivid, it has not only been preferred by writers, but also welcomed by the general public. Puns bring ease to serious literary works, make advertising words catchy, and add laughter to daily life. According to Colins Cobuild English Dictionary, "A pun is a clever and amusing use of a word with more than one meaning, or a word that sounds like another word, so that what you say has two different meanings." In Shorter Oxford English Dictionary, the pun is defined as the humorous use of a word in such a way as to suggest two or more meanings, or of words of similar sound with different meanings. Pun, a concise way to express humor and wit, is particularly favored by advertisers to enhance the appeal of language and thus influence the audiences' buying decision, then it can produce the following pragmatic functions in English advertising. 


\subsection{Humorous Effect}

Puns in English humor bring dexterity and vitality to the English language, in a variety of ways, too numerous to enumerate. Puns, as a commonly used technique in English rhetoric, are implicit, euphemistic, lively, witty and interesting, and then it has a lot of aftertaste. Understanding and mastering the rhetorical device of pun can not only make people feel the charm of English language expression, but also further increase the fun of English learning and improve learners' understanding and appreciation of English.

"Try our sweet corn. You' 11 smile from ear to ear." This is a commercial for sweet corn, and ear means "ear" and "ear". The idiom "from ear to ear" means not only to be happy from ear to ear because of satisfaction, but also to eat one ear after another because you like it. The puns in the advertising words are ingenious, novel and chic, unforgettable, produce humor, and receive excellent publicity results. At the same time, it is also benefit for the audience, short and concise advertising words reduce the time for the audience to input information and process information and it's easier for audience to remember.

\subsection{Stimulate Consumption}

The simulation of pun repair can use one word or one sentence to express two different meanings in order to make the language humorous and interesting, or to take advantage of this to get the effect of one and the other.

"Every Kid Should Have an Apple after School." In this advertisement, the word "apple" refers to a computer with a fruit and brand name. Usually, American children eat snacks, such as fruit and pastries, after school in the afternoon. Therefore, the literal meaning is to encourage children to eat more apples after school. The moral is to use the name of the personal computer. Apples are conducive to the physical and mental development of children. As a result, the clever use of "apple" has undoubtedly spurred the idea of parents buying an Apple computer for their children.

\subsection{Attracting Public Attention}

The use of the puns in advertisements can greatly increase the value of attention and memory. Since understanding puns is similar to guessing riddles, it needs to take a long processing time, so the puns can attract the attention of the audience for a period of time. They will remember it once they understand it. Some people may not be able to understand it for a while, they will continue to think until they find the answer, and they may ask other people, which will play the role of publicity. Understanding the pun, getting an intellectual satisfaction and thereby increasing the audience's recognition to the product, which is one of the effects of the advertisements? The use of puns in advertising can meet the psychological needs of people's aesthetic feeling. Pun is the flower of the wisdom of language. The use of pun can make the language vivid, witty and lively, humorous, meaningful and thought-provoking, shining with the brilliance of language wisdom, and making the human mind have a pleasant feeling. Give people an endless aftertaste of the enjoyment of the beauty of the language.

"What will the world be like without association?" The original meaning of the word association refers to a kind of imagination from one side to another. Advertising is a pun, emphasizing that if human beings lose the brand of "association", just as they lose imagination and creativity, the world will be unimaginable. Successfully embedded their own brand in the advertising language, and stressed that human beings can't lose association. After careful taste, people can't help but admire its creativity; people can't help but aftertaste.

\subsection{Passing on a Message}

This function is the most basic and important function of advertising puns, because an advertisement must convey a message to people. Without this feature, advertising will be a failure and meaningless. This function gives the mood of mass entertainment, but also stimulates the desire to buy and meet the needs of the public. Because it also contains information about products and services. In short, the public can get a wealth of information from advertising puns through short 
advertisements and advertisers try to convince them that this product can meet their needs and improve their quality of life.

"Nothing is beyond REACH." This is also a very classic advertisement, the clever use of the word "Reach" makes this sentence meaningful. If "Reach" is understood as a word, it means that the toothbrush is "easy to reach". But in fact, "Reach" is just the brand name of a toothbrush. The essence of this advertisement uses the word "Reach" to summarize the product and its main features.

\section{Pragmatic Function of Puns in English Advertising Violated the Cooperative Principle}

Sometimes, some of the slogans in English advertising will deliberately violate the principle of conversational cooperation, so as to achieve a certain pragmatic effect. However, puns in advertising language which violated the principle of cooperation has in the various norms of humor, novel, eyecatching, thought-provoking pragmatic functions, to achieve the advertising value to stimulate consumer interest and purchase desire. The followings are some examples:

"OIC" is an advertisement for a kind of glass. The amount of information provided in this glass advertising is very simple, obviously it violate the quantitative maxim, but it is very compelling, and makes people think. The audience uses the "measure of the criteria" to derive the conversational meaning of the advertising: this is a homophonic pun, the harmonic "Oh, I see." means I can see clearly with this glasses.

"We know eggsactly how to sell eggs". "eggsactly" was misspelling and should be "exactly", obviously the advertising violates the qualitative maxim. But this harmonic pun can attract the audience's special attention and deepen the impression.

"Less bread. No jam". The first thing that makes you think of a food advertisement about bread and sauce is a deliberate violation of the "relation maxim", and the use of homogenous puns creates another layer of conversational meaning. "Bread" refers to money, and "jam" refers to traffic congestion. So audiences will follow the relationship guidelines to say that if you ride the London Underground, you can save money and avoid traffic congestion.

"Which larger can claim to be truly German? This can." In this advertising, the grammar pun "can" has two grammatical functions so that it can cause ambiguity. This violates the "manner maxim", but on the other hand it can attract the attention of the audience, and thus derives the deep conversational meaning of this "filling" beer as "can" be truly called German beer.

\section{Summary}

Why is the pun so popular with advertisers and audiences? The reason is the pun has four significant effects: novel, economic, emotional and memory value. First of all, the pun designer is clever, catchy and amazing. Second, the pun is economic, timely and saves the advertisers' costs. At the same time, the pun can achieve emotional interaction, deliver the love, impress the audience, and achieve the persuasive purpose of advertising. Finally, understanding the pun can get a intellectual satisfaction, and increase the memory value of the audience. For the advertisers, it is necessary to make the dual contexts according to the expected goals. Based on the effect of the puns, the advertisers should reduce the degree of difficulty of deciphering, and make it easier for the audience to understand. For the audience, they need to decode the puns, integrate the two meanings, gain a overall understanding of the puns and then to achieve the purpose of the puns intended by the advertising designer. Although the audience has to pay a lot of time and mental labor in the process, because of the peculiar creativity of the puns, not only will the audience not be disgusted, but the audience will enjoy the process. While understanding puns requires additional cognitive effort on the part of the audience, advertisers. But because of its simplicity, vivid, humor, it is widely used in advertising, so that engage audiences and stimulate consumption to achieve the value of the goods widely and more effectively . 


\section{Acknowledgements}

This paper is supported by the Provincial departmental-level Scientific Research of Shaan xi province: Psycholinguistic Empirical Study on the Restaurant Name in Xi'an Provence from the Perspective of Cultural Differences. Project Number:19JK0625.

\section{References}

[1]. Grice, H. Logic and Conversation[M]. New York: Academic Press, 1975.

[2]. Oxford Advanced Learner's English - Chinese Dictionary [Z], Oxford University Press.1997/7, P1202.

[3]. Cui Gang. English Advertisement 300 Sentences [M]. Beijing: Beijing Polytechnic University Press. 2014.

[4]. Mullen, Puns, Relevance and Appreciation in Advertisements [J]. Journal of Pragmatic, 2005 (37: 707-721). 